\title{
Dermatopharmacokinetic Studies in India: A Review
}

Rahul Mayee $^{1 *}$ and Swati Rawat ${ }^{2}$

${ }^{1}$ MGM School of Biomedical Sciences, Aurangabad (MS), India

${ }^{2}$ Bhagawan College of Pharmacy, Aurangabad (MS), India

\begin{abstract}
The purpose of this study involves research to assess the bioavailability studies in India by Dermatopharmacokinetic method. The studies were conducted following many of the Indian pharmacy markets performance. A washout period of two days was kept between the two periods of the study. It was mainly focused on the amount of research specifically oriented towards the Dermatopharmacokinetic research in India.
\end{abstract}

Keywords: Dermatopharmacokinetic studies; Dermal Microdialysis; FDA

\section{Introduction}

Bioequivalence studies in India, though the major break-though, in the Indian Pharma- growth and about a decade old, has not yet penetrated equally as far as the dermal products are concerned. Like many other dosage forms, those getting absorbed through various routes, the dermal preparations which are absorbed from skin, also contributes the respectable market performance [1]. But unlike for other dosage forms the bioavailability or bioequivalence studies, for dermal preparations are very less studied.

Many regulatory bodies including US FDA are feeling it necessary to set up the guidelines for the BA BE studies of the dermal preparations. Accordingly, FDA had already published the draft guideline for this purpose. Though these guidelines are still not for the commercial purpose, but hold the greatest interest for academic research, where in, the use and importance of Dermatopharmacokinetic studies, could be verified [2].

Skin Stripping and Skin Microdialysis and two well verified methods for the dermatopharmacokinetic studies so far [3]. But the number of drugs verified by these methods is still very few. Large number of classes of drugs is available under the title Topical Preparations [4]. The scientists working on Novel Drug Delivery System research are also concentrating on the Drug patches or Under-skin Depot drug delivery system. With considerable amount of research going on to engineer the new drug delivery systems through skins, even for the potent drugs like hormones, the amount of research specifically oriented towards the Dermatopharmacokinetic research of sadly less.

Many research trials in this area have proved that the dermal penetration of any drug is governed mainly by the formulation contents and the skin barrier [5]. Thus it could be easily predicted that rate and the extent of the absorption and elimination of a drug in different people with much different skin type will be different. Thus the dermatopharmacokinetic studies could predict more efficiently which type of vehicle or the formulation ingredients are needed for a particular geographical area depending upon the skin type of the population in that particular area.

The research leading to dermal-way of drug delivery is one of the potent areas of interest of many scientists of Pharmaceutical industries, for the major reasons like safety promised and the patient acceptability. Dermal dosage forms are one of the safest drug delivery devices with minimal side effects [6]. Any untoward incidence if observed the patient or recipient could be almost immediately cut-off from receiving the further dosage by simply removing the drug dosage form applied, which in case of other dosage forms is more difficult or even impossible. Few though not all dosage forms need the expert personnel to administer the drug in patient's body, dermal products could be applied with ease by the patient him or herself [7].

ICH E6 guidelines for the bioequivalence studies are very elaborate as far as methodologies expected to be adopted for conducting these studies. The conventional bioequivalence studies are conducted by measuring the drug concentrations in biological fluids like blood or urine. The dermal products deliver the drug in the body through the skin as barrier and it is the main matrix that governs the rate and extends of absorption of the drug [8]. This is the need of the day to research upon and construct the solid scientific foundation of methodologies, which in turn will be acceptable by all the regulatory authorities to verify the formulation effect, therapeutic and Pharmacokinetic equivalence for the new upcoming dermal dosage forms.

The need of Dermatopharmacokinetic studies is needed to be cross-verified. The widely used skin stripping method and or Dermal Microdialysis should be tested on various population samples, to cross verify its race/location dependency [9]. These studies are needed to be part of the regulatory requirements for getting the marketing authorization for the dermal products.

\section{References}

1. Mary BE (2005) 'Defining the topical generic market'. SFBC Anapharm symposium.

2. Herkenne C, Naik A, Kalia YN, Hadgraft J, Guy RH (2006) Pig ear skin ex vivo as a model for in vivo dermatopharmacokinetic studies in man. Pharm Res 23 : 1850-1856.

3. Lodén $M$, Akerström U, Lindahl K, Berne B (2004) Bioequivalence determination of topical ketoprofen using a dermatopharmacokinetic approach and excised skin penetration. Int J Pharm 284: 23-30.

4. Walter WH (2005) Bioequivalence studies of topical preparations: statistical considerations.

5. Benfeldt E, Hansen SH, Volund A, Menne T, Shah VP (2007) Bioequivalence of topical formulations in humans: evaluation by dermal microdialysis sampling and the dermatopharmacokinetic method. J Invest Dermatol 127: 170-178.

*Corresponding author: Rahul Mayee, MGM School of Biomedical Sciences, Aurangabad (MS), India, E-mail: rahul.mayee@rediffmail.com

Received June 25, 2012; Published October 30, 2012

Citation: Mayee R, Rawat S (2012) Dermatopharmacokinetic Studies in India: A Review. 1:427. doi:10.4172/scientificreports.427

Copyright: ( 2012 Mayee R, et al. This is an open-access article distributed unde the terms of the Creative Commons Attribution License, which permits unrestricted use, distribution, and reproduction in any medium, provided the original author and source are credited. 
6. Pershing LK, Bakhtian S, Poncelet CE, Corlett JL, Shah VP (2006) Comparison of skin stripping, in vitro release, and skin blanching response methods to measure dose response and similarity of triamcinolone acetonide cream strengths from two manufactured sources.

7. Pershing LK, Nelson JL, Corlett JL, Shrivastava SP, Hare DB, et al. (2003) Assessment of dermatopharmacokinetic approach in the bioequivalence determination of topical tretinoin gel products. J Am Acad Dermatol 48: 740751.
8. US. Department of Health and Human Services (1998) Guidance for Industry, Topical Dermatological Drug Product NDAs and ANDAs - In Vivo Bioavailability, Bioequivalence, In Vitro Release, and Associated Studies DRAFT GUIDANCE. Food and Drug Administration, Center for Drug Evaluation and Research (CDER)

9. Evaluation of Topical Bioavailability in Human Stratum Corneum in Vivo by Tape Stripping Using a Direct Spectroscopic Method. Fässler Tassopoulos Inaugural dissertation. 